\title{
FREE VIBRATIONS SPECTRUM OF PERIODICALLY INHOMOGENEOUS RAYLEIGH BEAMS USING THE TOLERANCE AVERAGING TECHNIQUE
}

\author{
Marcin Świątek, Łukasz Domagalski, JarosŁaw Jędrysiak \\ Lodz University of Technology, Department of Structural Mechanics, Eódź, Poland \\ e-mail:marcin.swiatek@dokt.p.lodz.pl; lukasz.domagalski@p.lodz.pl; jarek@p.lodz.pl
}

\begin{abstract}
In this paper, linear-elastic Rayleigh beams with a periodic structure are considered. Dynamics of such beams is described by partial differential equations with non-continuous highly oscillating coefficients. The analysis of dynamic problems using the aforementioned equations is very often problematic to perform. Thus, other simplified models of Rayleigh beams are proposed. Some of these models are based on the concept of the effective stiffness. Among them, one can distinguish the theory of asymptotic homogenization. However, in these models, the size of the mesostructure parameter (the size of a periodicity cell) is often neglected. Therefore, a non-asymptotic averaged model of the periodic beam is introduced, called the tolerance model, which is derived by applying the tolerance averaging technique (TA). The obtained tolerance model equations have constant coefficients, and in contrast to other averaged models, some of them depend on the size of the periodicity cell.
\end{abstract}

Keywords: periodicity cell, Rayleigh beam, tolerance averaging technique

\section{Preface}

Beams are the simplest representations of periodic structures. Numerous examples of engineering applications, for instance in acoustic isolations, are the main reason for interest in such objects. In such beams, one can distinguish a small repetitive element called the periodicity cell. Periodic objects can represent approximate models of some complex systems.

Propagation of the elastic wave and linear vibrations in periodic beams are considered in many papers. Vibration band gaps were investigated by Xiang and Shi (2009) by the differential quadrature method. A comprehensive research on inhomogeneous beams vibrations was presented by Hajianmaleki and Qatu (2013). The transfer matrix method, adapted in analysis of flexural wave propagation in a beam on an elastic foundation and in investigating natural frequencies of non-uniform beams, can be found in Yu et al. (2012) and Xu et al. (2016), respectively. Wave propagation in beams with periodically varying stiffness is considered in Chen (2013) by the use of the multireflection method. In this paper, linear-elastic Rayleigh beams with a periodic structure are considered. Dynamics of such beams is described by partial differential equations with non-continuous highly oscillating coefficients. The analysis of dynamic problems using the aforementioned equations is very often problematic to perform. Thus, other simplified models of Rayleigh beams are proposed. Some of these models are based on the concept of the effective stiffness. Among them, one can distinguish the theory of asymptotic homogenization introduced in works by Kohn and Vogelius (1984), Papanicolau et al. (1978), Bakhvalov and Panasenko (1989), Sánchez-Palencia (1980) and Zhikov et al. (1994). The microperiodic beam equilibrium equations in frames of the homogenization theory were studied by Kolpakov (1991, 1998, 1999). However, in governing equations of these models, the size of the mesostructure parameter (the size of the periodicity cell) is often neglected. Therefore, a non-asymptotic averaged model of the periodic beam is introduced. This model is called the tolerance model and is 
derived by applying the tolerance modelling technique, c.f. Woźniak et al. (2008), Awrejcewicz (2010), Woźniak and Wierzbicki (2000). The obtained tolerance model equations have constant coefficients and, in contrast to other averaged models, some of them depend on the size of the periodicity cell. The proposed method can be adopted to any differential equations with highly oscillating coefficients. The suggested approach, in contrast to the asymptotic homogenization, enables analysis of the mesostructure size. The method found numerous applications in structural mechanics. Macro-dynamics of microperiodic elastic beams was analysed by Mazur-Śniady (1993). Geometrically nonlinear vibrations of slender mesoperiodic beams were investigated in the paper by Domagalski and Jędrysiak (2016). The method was widely applied in the analysis of microstructured plates: thin plates with an elastic periodic foundation, Jędrysiak (2003), honeycomb lattice-type plates, Cielecka and Jędrysiak (2006), geometrically nonlinear thin plates, Domagalski and Jędrysiak (2015) and thin functionally graded plates, Jędrysiak (2013, 2014) and Kaźmierczak and Jędrysiak (2011). The TA technique was also applied in plates stability problems, cf. Jędrysiak (2000) and Jędrysiak and Michalak (2011). The tolerance averaging technique was also applied in the analysis of wavy type plates Michalak (2001) and many other engineering problems.

In this paper, a new tolerance model of a Rayleigh beam with weakly slowly-varying functions is proposed Tomczyk (2013), Jędrysiak (2017). Natural boundary conditions are also obtained and presented for a newly derived tolerance model. The presented tolerance model equations are used to determine natural vibration frequencies and natural forms of vibrations. Solutions obtained from the proposed model are compared with those corresponding to the finite element model. The paper is arranged as follows: basic assumptions of the inhomogeneous Rayleigh beams are presented in Section 2. The elemental and essential basis of the tolerance averaging technique are quoted in Section 3. The main model equations for examples considered in this paper are derived in Section 4. The numerical methods of solution, validation of the model, final results and comparison with the finite element method are presented in Section 5. Finally, the discussion and conclusions are given in Section 6.

\section{Formulation of the problem}

A beam made of a linear-elastic material, associated with a three-dimensional Cartesian coordinate system $O x y z$ is considered. The beam axis is collinear with the $x$-axis of the local coordinate system. The problem can be treated as one-dimensional, so that there is defined a region $\Omega \equiv[0, L]$ occupied by the beam, where $L$ is the beam length. The considered beam consists of many repetitive elements, called periodicity cells. The basic cell is defined as $\Delta \equiv[-l / 2, l / 2]$,

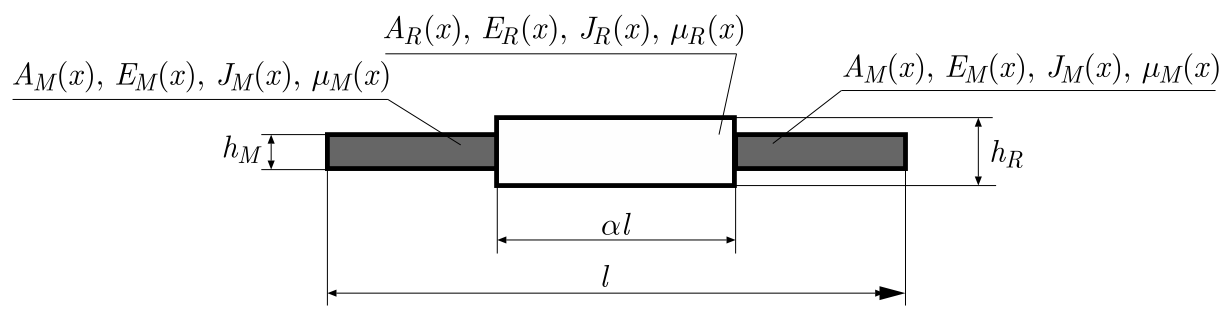

Fig. 1. A periodicity cell

where $l \ll L$ is length of the cell and is named the mesostructure parameter. The following denotations are introduced: lateral deflection $w=w(x, t)$, lateral stiffness $E J=E(x) J(x)$, mass per unit length $\mu=\mu(x)$, rotational moment of inertia per unit length $\vartheta=\vartheta(x)$ and transverse load $q=q(x, t)$. Furthermore, let $\partial^{k}=\partial^{k} / \partial x^{k}$ be the $k$-th derivative of a function taken with respect to the $x$ coordinate, and the overdot stands for the derivative taken with respect to time. 
Thus, the strain and kinetic energy of the beam can be described in the following form

$$
\mathcal{W}=\frac{1}{2} E J \partial^{2} w \partial^{2} w \quad \mathcal{K}=\frac{1}{2} \mu \dot{w} \dot{w}+\frac{1}{2} \vartheta \partial \dot{w} \partial \dot{w}
$$

The Lagrangian function $\mathcal{L}=\mathcal{L}\left(x, t, w, \dot{w}, \partial \dot{w}, \partial^{2} w\right)$ is defined as

$$
\mathcal{L}=\mathcal{W}-\mathcal{K}-q w
$$

The equations of motion are given by Hamilton's principle

$$
\delta \mathcal{A}=\delta \int_{t_{0}}^{t_{1}} \int_{0}^{L} \mathcal{L} d x d t=\int_{t_{0}}^{t_{1}} \int_{0}^{L} \delta \mathcal{L} d x d t=0
$$

After some common variation calculus operations, the equation of motion of the Rayleigh beam with highly oscillating non-continuous coefficients is obtained

$$
\partial^{2}\left(E J \partial^{2} w\right)+\mu \ddot{w}-\partial(\vartheta \partial \ddot{w})=q
$$

\section{Tolerance modelling}

\subsection{Preliminary notions}

The main objective of this paper is to propose a new averaged model of the Rayleigh beam. This new approach is based on the concept of weakly slowly-varying functions. The averaged equations of the periodic beam are derived using the tolerance modelling technique. The fundamental concepts of the tolerance modelling approach - tolerance relations, slowly-varying functions $(S V)$, tolerance periodic functions $(T P)$, fluctuation shape functions (FSFs) and averaging operation, are outlined in the monographs by Woźniak and Wierzbicki (2000), Woźniak et al. (2008), Awrejcewicz (2010). There are introduced the following denotations: $\Delta(x) \equiv x+\Delta$, $\Omega_{\Delta} \equiv\{x \in \Omega: \Delta(x) \in \Omega\}, x \in R^{m}$. Subsequently, a subset $\Delta$ of $R^{m}$ is called the periodicity cell with $l$ as a cell dimension. Every cell $\Delta(x), x \in \Omega_{\Delta}$, refers to the cell in $\Omega$ with the center at $x$. The averaging operator for an arbitrary integrable function $f$ is defined by

$$
\langle f\rangle(x)=\frac{1}{l} \int_{\Delta(x)} f(y) d y \quad x \in \Omega_{\Delta} \quad y \in \Delta(x)
$$

The micro-macro decomposition is a fundamental operation of the tolerance averaging technique. It states that the transverse deflection of the beam $w(x, t)$ (unknown of the partial differential equations describing behavior of the microheterogeneous structure) can be decomposed into: the unknown averaged displacement $W(x, t)$ (a weakly slowly-varying function in the periodicity direction) and the highly oscillating fluctuation of the displacement, represented by the known highly oscillating $\Delta$-periodic fluctuation shape function $h^{A}(x)$ multiplied by the unknown fluctuation amplitude $V^{A}(x, t)$ - weakly slowly-varying $(W S V)$ in the periodicity direction. In this case, the micro-macro decomposition becomes

$$
w(x, t)=W(x, t)+h^{A}(x) V^{A}(x, t) \quad A=1, \ldots, N \quad W(\cdot), V^{A}(\cdot) \in W S V_{d}^{2}(\Omega, \Delta)
$$

From now, $W(x, t)$ is a new basic kinematic unknown and $V^{A}(x, t)$ is an additional kinematic unknown. The uppercase integer states that the unknown functions are assumed to be weakly slowly-varying up to the second derivative order. The function $F(\cdot)$ will be referred to as the 
weakly slowly-varying with respect to the cell $\Delta$ and the tolerance given by $\delta \equiv\left(\alpha, \delta_{0}, \delta_{1}, \ldots, \delta_{R}\right)$, if and only if the following condition is satisfied

$$
\exists(x, y) \in \Omega^{2} \quad\left[(x \stackrel{\alpha}{\approx} y) \Rightarrow F(x) \stackrel{\delta_{0}}{\approx} F(y) \wedge \partial^{k} F(x) \stackrel{\delta_{k}}{\approx} \partial^{k} F(y), k=1,2, \ldots, R\right]
$$

where $\partial^{0} F(\cdot) \equiv F(\cdot)$.

Under the above conditions, it can be written $F \in W S V_{\delta}^{R}(\Omega, \Delta)$. In the applications of the tolerance modelling, the tolerance parameter $\alpha=l$ is known a priori as a certain mesostructure length, whereas values of the tolerance parameters $\delta_{0}, \delta_{1}, \ldots, \delta_{R}$ can be determined only a posteriori, i.e. after obtaining a solution to the considered initial-boundary value problem. The highly oscillating fluctuation shape functions $h^{A}$ are postulated a priori in every problem under consideration and describe the unknown fields oscillations caused by the structure inhomogeneity. Apart from the restriction of $l$-periodicity, the FSFs have to satisfy the following conditions

$$
\begin{array}{lrl}
\left\langle\mu h^{A}\right\rangle & =0 \quad\left\langle\mu h^{A} h^{B}\right\rangle=0 \quad \text { for } \mathrm{A} \neq B \\
\partial^{m} h^{A} & \in O\left(l^{2-m}\right) & A, B=1, \ldots, N
\end{array}
$$

Another assumption is the tolerance averaging approximation. For the purposes of this article, the following denotations are introduced. Let $e, f \in L_{l o c}^{2}(R)$ be the known l-periodic functions and let $F \in W S V_{d}^{1}(0, L), d \equiv\left(l, \delta_{0}, \delta_{1}\right)$. By the tolerance averaging of $e F+f \partial_{1} F$ is meant $\left\langle e F+f \partial_{1} F\right\rangle_{T}(x) \equiv\langle e\rangle F(x)+\langle f\rangle \partial_{1} F(x)$ for every $x \in(l / 2, L-l / 2)$. The tolerance averaging approximation is an approximation of $\left\langle e F+f \partial_{1} F\right\rangle(x)$ by $\left\langle e F+f \partial_{1} F\right\rangle_{T}(x)$ for every $x \in(l / 2, L-l / 2)$. Thus, the tolerance averaging approximation has the form

$$
\left\langle e F+f \partial_{1} F\right\rangle(x)=\left\langle e F+f \partial_{1} F\right\rangle_{T}(x)+O(l) \quad d \equiv\left(l, \delta_{0}, \delta_{1}\right)
$$

where $e(\cdot), f(\cdot)$ are the known functions and $F(\cdot)$ is unknown in the initial-boundary value problem under consideration.

\subsection{The averaged model equations}

The averaging operation is performed, after substituting micro-macro decomposition (3.2) into Lagrangian (2.2). Thus, the variation of the averaged action functional can be written as

$$
\delta \mathcal{A}=\delta \int_{t_{0}}^{t_{1}} \int_{0}^{L}\left\langle\mathcal{L}_{h}\right\rangle d x d t=\int_{t_{0}}^{t_{1}} \int_{0}^{L} \delta\left\langle\mathcal{L}_{h}\right\rangle d x d t=0
$$

Knowing that

$$
\begin{aligned}
& -\kappa=\partial^{2} w=\partial^{2} W+\partial^{2}\left(h^{A} V^{A}\right)=\partial^{2} W+\partial\left(\partial h^{A} V^{A}+h^{A} \partial V^{A}\right) \\
& =\partial^{2} W+\partial^{2} h^{A} V^{A}+2 \partial h^{A} \partial V^{A}+h^{A} \partial^{2} V^{A} \\
& -\delta \kappa=\partial^{2} \delta W+\partial^{2} h^{A} \delta V^{A}+2 \partial h^{A} \partial \delta V^{A}+h^{A} \partial^{2} \delta V^{A} \\
& -M=E J \partial^{2} w=E J\left(\partial^{2} W+\partial^{2} h^{B} V^{B}+2 \partial h^{B} \partial V^{B}+h^{B} \partial^{2} V^{B}\right) t
\end{aligned}
$$

the Lagrangian variation is

$$
\delta \mathcal{L}=\delta \mathcal{W}-\delta \mathcal{K}-q \delta w=M \delta \kappa-\mu \dot{w} \delta \dot{w}-\vartheta \partial \dot{w} \partial \delta \dot{w}-q \delta w
$$

Finally

$$
\int_{t_{0}}^{t_{1}} \int_{0}^{L} \delta \mathcal{K} d x d t=\int_{t_{0}}^{t_{1}} \int_{0}^{L}(\mu \ddot{w} \delta w+\vartheta \partial \ddot{w} \partial \delta w) d x d t
$$


and

$$
\delta \mathcal{L}=M \delta \kappa+(\mu \ddot{w}-q) \delta w+\vartheta \partial \ddot{w} \partial \delta w
$$

Let micro-macro decomposition (3.2) be substituted into the components of the Lagrangian and averaged over a periodicity cell. It should be noted that

$$
\delta w=\delta W+h^{A} \delta V^{A}
$$

The variation of the averaged bending energy gives

$$
\langle\delta \mathcal{L}\rangle=\langle M \delta \kappa\rangle=\langle M\rangle \partial^{2} \delta W+\left\langle M \partial^{2} h^{A}\right\rangle \delta V^{A}+2\left\langle M \partial h^{A}\right\rangle \partial \delta V^{A}+\left\langle M h^{A}\right\rangle \partial^{2} \delta V^{A}
$$

where

$$
\begin{aligned}
& \langle M\rangle=\langle E J\rangle \partial^{2} W+\left\langle E J \partial^{2} h^{B}\right\rangle V^{B}+2\left\langle E J \partial h^{B}\right\rangle \partial V^{B}+\left\langle E J h^{B}\right\rangle \partial^{2} V^{B} \\
& \left\langle M \partial^{2} h^{A}\right\rangle=\left\langle E J \partial^{2} h^{A}\right\rangle \partial^{2} W+\left\langle E J \partial^{2} h^{A} \partial^{2} h^{B}\right\rangle V^{B}+2\left\langle E J \partial^{2} h^{A} \partial h^{B}\right\rangle \partial V^{B} \\
& \quad+\left\langle E J \partial^{2} h^{A} h^{B}\right\rangle \partial^{2} V^{B} \\
& \left\langle M \partial h^{A}\right\rangle=\left\langle E J \partial h^{A}\right\rangle \partial^{2} W+\left\langle E J \partial h^{A} \partial^{2} h^{B}\right\rangle V^{B}+2\left\langle E J \partial h^{A} \partial h^{B}\right\rangle \partial V^{B}+\left\langle E J \partial h^{A} h^{B}\right\rangle \partial^{2} V^{B} \\
& \left\langle M h^{A}\right\rangle=\left\langle E J h^{A}\right\rangle \partial^{2} W+\left\langle E J h^{A} \partial^{2} h^{B}\right\rangle V^{B}+2\left\langle E J h^{A} \partial h^{B}\right\rangle \partial V^{B}+\left\langle E J h^{A} h^{B}\right\rangle \partial^{2} V^{B}
\end{aligned}
$$

The total variation of the Lagrangian is

$$
\begin{aligned}
\delta \mathcal{L} & =\langle M\rangle \partial^{2} \delta W+\left(\langle\vartheta\rangle \partial \ddot{W}+\left\langle\vartheta \partial h^{A}\right\rangle \ddot{V}^{A}\right) \partial \delta W+\left(\langle\mu\rangle \ddot{W}+\left\langle\mu h^{A}\right\rangle \ddot{V}^{A}-\langle q\rangle\right) \delta W \\
& +\left(\left\langle M \partial^{2} h^{A}\right\rangle+\left\langle\mu h^{A}\right\rangle \ddot{W}+\left\langle\mu h^{A} h^{B}\right\rangle \ddot{V}^{B}+\left\langle\vartheta \partial h^{A}\right\rangle \partial \ddot{W}\right. \\
& \left.+\left\langle\vartheta \partial h^{A} \partial h^{B}\right\rangle \ddot{V}^{B}-\left\langle q h^{A}\right\rangle\right) \delta V^{A}+2\left\langle M \partial h^{A}\right\rangle \partial \delta V^{A}+\left\langle M h^{A}\right\rangle \partial^{2} \delta V^{A}
\end{aligned}
$$

After some transformations

$$
\begin{aligned}
\delta \mathcal{L} & =\left[\partial^{2}\langle M\rangle-\partial\left(\langle\vartheta\rangle \partial \ddot{W}+\left\langle\vartheta \partial h^{A}\right\rangle \ddot{V}^{A}\right)+\langle\mu\rangle \ddot{W}+\left\langle\mu h^{A}\right\rangle \ddot{V}^{A}-\langle q\rangle\right] \delta W \\
& +\left(\left\langle M \partial^{2} h^{A}\right\rangle-2 \partial\left\langle M \partial h^{A}\right\rangle+\left\langle\mu h^{A}\right\rangle \ddot{W}+\left\langle\mu h^{A} h^{B}\right\rangle \ddot{V}^{B}+\left\langle\vartheta \partial h^{A}\right\rangle \partial \ddot{W}\right. \\
& \left.+\left\langle\vartheta \partial h^{A} \partial h^{B}\right\rangle \ddot{V}^{B}+\partial^{2}\left\langle M h^{A}\right\rangle-\left\langle q h^{A}\right\rangle\right] \delta V^{A}+\partial(\langle M\rangle \partial \delta W) \\
& +\partial\left[\left(\langle\vartheta\rangle \partial \ddot{W}+\left\langle\vartheta \partial h^{A}\right\rangle \ddot{V}^{A}-\partial\langle M\rangle\right) \delta W\right] \\
& +\partial\left(\left\langle M h^{A}\right\rangle \partial \delta V^{A}\right)-\partial\left[\left(\partial\left\langle M h^{A}\right\rangle-2\left\langle M \partial h^{A}\right\rangle\right) \delta V^{A}\right]
\end{aligned}
$$

This leads to a system of differential equations

$$
\begin{aligned}
& \delta W: \quad \partial^{2}\langle M\rangle-\partial\left(\langle\vartheta\rangle \partial \ddot{W}+\left\langle\vartheta \partial h^{A}\right\rangle \ddot{V}^{A}\right)+\langle\mu\rangle \ddot{W}+\left\langle\mu h^{A}\right\rangle \ddot{V}^{A}-\langle q\rangle=0 \\
& \delta V^{A}: \quad\left\langle M \partial^{2} h^{A}\right\rangle-2 \partial\left\langle M \partial h^{A}\right\rangle+\left\langle\mu h^{A}\right\rangle \ddot{W}+\left\langle\mu h^{A} h^{B}\right\rangle \ddot{V}^{B} \\
& \quad+\left\langle\vartheta \partial h^{A}\right\rangle \partial \ddot{W}+\left\langle\vartheta \partial h^{A} \partial h^{B}\right\rangle \ddot{V}^{B}+\partial^{2}\left\langle M h^{A}\right\rangle-\left\langle q h^{A}\right\rangle=0
\end{aligned}
$$

and natural boundary conditions

$$
\begin{aligned}
& \left.\left(\langle\vartheta\rangle \partial \ddot{W}+\left\langle\vartheta \partial h^{A}\right\rangle \ddot{V}^{A}-\partial\langle M\rangle\right) \delta W\right|_{0} ^{L}+\left.\langle M\rangle \partial \delta W\right|_{0} ^{L}+\left.\left\langle M h^{A}\right\rangle \partial \delta V^{A}\right|_{0} ^{L} \\
& \quad+\left.\left(\partial\left\langle M h^{A}\right\rangle-2\left\langle M \partial h^{A}\right\rangle\right) \delta V^{A}\right|_{0} ^{L}=0
\end{aligned}
$$


The $N+1$ differential equations for the macro-deflection and its fluctuation amplitudes are

$$
\begin{aligned}
& \partial^{2}\langle M\rangle-\langle\vartheta\rangle \partial^{2} \ddot{W}-\left\langle\vartheta \partial h^{A}\right\rangle \partial \ddot{V}^{A}+\langle\mu\rangle \ddot{W}+\left\langle\mu h^{A}\right\rangle \ddot{V}^{A}-\langle q\rangle=0 \\
& \left\langle M \partial^{2} h^{A}\right\rangle-2 \partial\left\langle M \partial h^{A}\right\rangle+\left\langle\mu h^{A}\right\rangle \ddot{W}+\left\langle\mu h^{A} h^{B}\right\rangle \ddot{V}^{B}+\left\langle\vartheta \partial h^{A}\right\rangle \partial \ddot{W} \\
& \quad+\left\langle\vartheta \partial h^{A} \partial h^{B}\right\rangle \ddot{V}^{B}+\partial^{2}\left\langle M h^{A}\right\rangle-\left\langle q h^{A}\right\rangle=0
\end{aligned}
$$

The weight-averaged bending moments have the following form

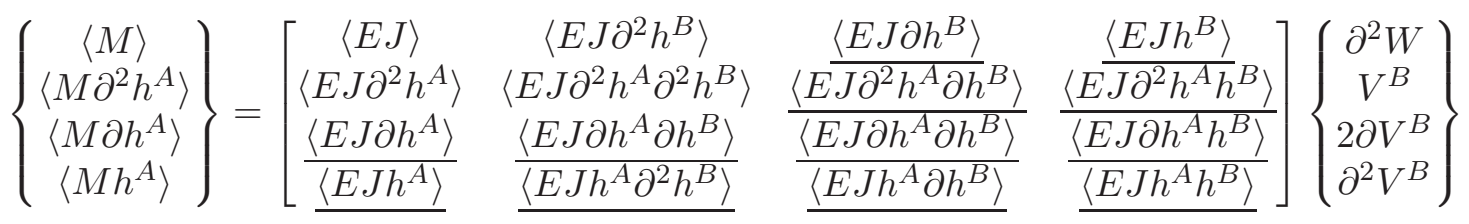

where $W(x, t), V^{A}(x, t)$ and their derivatives are the new kinematic unknowns. Together with the averaged equation of motion, the following natural boundary conditions (for $x=0, L$ ) with averaged coefficients are obtained

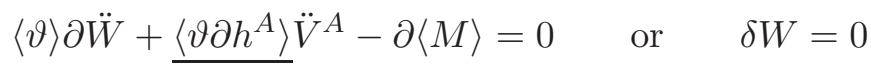

$$
\begin{aligned}
& \langle M\rangle=0 \quad \text { or } \quad \partial \delta W=0 \\
& \partial \underline{\left\langle M h^{A}\right\rangle}-2 \underline{\left\langle M \partial h^{A}\right\rangle}=0 \quad \text { or } \quad \delta V^{A}=0 \\
& \left\langle M h^{A}\right\rangle=0 \quad \text { or } \quad \partial \delta V^{A}=0
\end{aligned}
$$

It is worth mentioning that expressions (3.20) reduce to classic natural boundary conditions for a homogeneous beam (Fung, 1965). Moreover, the underlined coefficients are dependent on the mesostructure size $l$. The external load is assumed to be zero in the analysis of natural vibrations of the beam.

\section{Examples of applications}

In this Section, the derived averaged model is adapted in a study of some special problems. The object under consideration is a simply supported beam with length $L$. The beam has a rectangular cross section and is made of some small repetitive elements. The periodicity cell, presented in Fig. 1, has a symmetrical shape and is divided into three segments. The segment material and geometrical properties may vary depending on each case.

One of the most significant components of the tolerance modelling is determination of the fluctuation shape functions. The fluctuation shape functions can be assumed as forms of eigenvibrations one the periodicity cell. In this model, FSFs are obtained from finite element analysis of the periodicity cell, although the common practice is to use approximate solutions such as $l$-periodic trigonometric functions.

In order to obtain a system of algebraic equations of motion, the Galerkin method is applied. The trial solutions are assumed in the form of truncated trigonometric series

$$
W(x, t)=\sum_{m=1}^{M_{w}} X_{m}(x) W_{m}(t) \quad V^{A}(x, t)=\sum_{n=1}^{M_{V}^{A}} Y_{m}^{A}(x) V_{m}^{A}(t) \quad A=1, \ldots, N
$$

where the weight functions $X_{m}$ and $Y_{m}^{A}$ are chosen to satisfy the boundary conditions of a simply supported beam

$$
X_{m}(x)=\sin \frac{m \pi x}{L} \quad Y_{m}^{A}= \begin{cases}\sin \frac{n \pi x}{L} & \text { for } \quad A \in E S F \\ \cos \frac{(n-1) \pi x}{L} & \text { for } \quad A \in O S F\end{cases}
$$


The functions $X_{m}$ and $Y_{m}^{A}$ satisfy the assumed boundary conditions at $x=0, L$, where ESF and $O S F$ stands for an even and odd fluctuation shape function respectively. The known relation is solved with respect to the unknown trial function coefficients (Zienkiewicz et al., 2013). The number of terms in the expansion results from the condition of convergence of the solution. In order to obtain the natural frequencies of the beam, the eigenproblem of the dynamic stiffness matrix is solved. In the numerical solutions, the size of the matrix is limited to the finite value

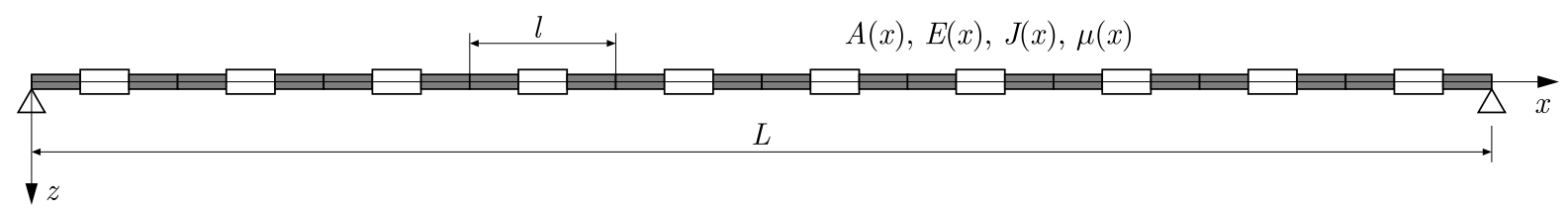

Fig. 2. The considered beam

\section{Results and discussion}

This Section is dedicated to the analysis of free vibrations of a Rayleigh beam. The beam has length $L=1.0 \mathrm{~m}$ and is composed of 10 periodicity cells with length $l=0.1 \mathrm{~m}$ and cross section width $b=0.01 \mathrm{~m}$. Three different beams with variable cross section height $h_{M}$, Young's modulus $E_{M}$ and mass density $\rho_{M}$ are analyzed. For each beam, there are considered three individual cases. The properties of the central periodicity cell segment - height $h_{R}=0.008 \mathrm{~m}$, Young's modulus $E_{R}=205 \mathrm{GPa}$ and density $\rho_{R}=7850 \mathrm{~kg} / \mathrm{m}^{3}$ are constant in all analyzed cases.

As an example, 3 cases: A, B and $\mathrm{C}$ are analyzed. For each case, one of the material or geometrical parameter of the periodicity cell has an individual value. The values of $h_{M}, E_{M}$ and $\rho_{M}$ parameters for all cases are presented in Table 1.

Table 1. Analyzed-cases

\begin{tabular}{|c|c|c|c|}
\hline Case & $h_{M}[\mathrm{~m}]$ & $E_{M}[\mathrm{GPa}]$ & $\rho_{M}\left[\mathrm{~kg} / \mathrm{m}^{3}\right]$ \\
\hline A1 & 0.004 & \multirow{6}{*}{205.000} & \\
\hline A2 & 0.005 & & 7850.00 \\
\hline$\overline{\mathrm{A} 3}$ & 0.006 & & \\
\hline $\bar{B} 1$ & \multirow{6}{*}{0.008} & & 3925.00 \\
\hline B2 & & & 1962.50 \\
\hline B3 & & & 981.25 \\
\hline $\mathrm{C} 1$ & & 102.500 & \multirow{3}{*}{7850.00} \\
\hline $\mathrm{C} 2$ & & 51.250 & \\
\hline C3 & & 25.625 & \\
\hline
\end{tabular}

In order to validate the tolerance model, a finite element method procedure is applied in Maple software. The finite element model is assembled with 30 Rayleigh beam elements with Hermitian polynomials and the consistent mass matrix. As a result, the model has 31 nodes with 62 degrees of freedom.

The natural frequencies, which are obtained using the tolerance averaging technique (TA) and the finite element method (FE), are compared in Table 2 . The validation of first 22 natural frequencies for cases A2, B2 and $\mathrm{C} 2$ for the mesostructure $\alpha=0.5$ is performed. The TA model results are presented as gray dots, and the FE model as black rings. The received values of frequencies are given in $[\mathrm{Hz}]$. The first five natural frequencies for cases A2, B2, C2 are listed in Table 2. $80.878 \mathrm{~Hz}, 147.330 \mathrm{~Hz}$, and $73.581 \mathrm{~Hz}$ are the least derived values of natural frequencies in cases A2, B2, and $\mathrm{C} 2$, respectively. The relative error does not exceed $2 \%$ for the first five 
frequencies. It is noticeable that the presented bandwidth is not entirely continuous and uniform. Among all obtained frequencies in Fig. 3, separated bands of frequencies can be observed the chains preceded and followed by some intervals. These interruptions in the bandwidth, highlighted with gray backgrounds, are called band gaps. In case A, the first band interval reveals between the 9th and 10th natural frequency. The difference between these frequencies arrives at $3133 \mathrm{~Hz}$. Another gap appears between the 20th and 21st natural frequency. In this case, the difference rises to $8454 \mathrm{~Hz}$. In case B, the gaps occur between the 10th, 11th and 20th, 21st free vibration frequencies, and the intervals in the bandwidth reach 7657 and $14164 \mathrm{~Hz}$, respectively. In case $\mathrm{C}$, the band gaps reveal at the same frequencies as in case $\mathrm{A}$, and the magnitudes of the interludes are $3663 \mathrm{~Hz}$ and $7171 \mathrm{~Hz}$, respectively.

Table 2. Natural frequencies for case A2, B2 and C2

\begin{tabular}{|c|c|c|c|c|c|c|c|c|c|}
\hline \multirow{2}{*}{$\omega_{i}$} & \multicolumn{3}{|c|}{$\mathrm{A} 2$} & \multicolumn{3}{c|}{$\mathrm{B} 2$} & \multicolumn{3}{|c|}{$\mathrm{C} 2$} \\
\cline { 2 - 10 } & $\mathrm{TA}$ & $\mathrm{FE}$ & $\frac{|\Delta \omega|}{\left|\omega^{F E}\right|}$ & $\mathrm{TA}$ & $\mathrm{FE}$ & $\frac{|\Delta \omega|}{\left|\omega^{F E}\right|}$ & $\mathrm{TA}$ & $\mathrm{FE}$ & $\frac{|\Delta \omega|}{\left|\omega^{F E}\right|}$ \\
\hline \hline$n$ & {$[\mathrm{~Hz}]$} & {$[\mathrm{Hz}]$} & {$[\%]$} & {$[\mathrm{Hz}]$} & {$[\mathrm{Hz}]$} & {$[\%]$} & {$[\mathrm{Hz}]$} & {$[\mathrm{Hz}]$} & {$[\%]$} \\
\hline 1 & 80.878 & 80.971 & 0.115 & 147.330 & 147.314 & 0.011 & 73.581 & 73.659 & 0.105 \\
\hline 2 & 322.607 & 324.108 & 0.463 & 589.309 & 589.054 & 0.043 & 293.269 & 294.534 & 0.430 \\
\hline 3 & 722.358 & 730.114 & 1.062 & 1325.846 & 1324.555 & 0.097 & 655.683 & 662.294 & 0.998 \\
\hline 4 & 1274.964 & 1300.213 & 1.942 & 2356.475 & 2352.406 & 0.173 & 1154.338 & 1176.231 & 1.861 \\
\hline 5 & 2043.391 & 2036.098 & 0.358 & 3679.510 & 3669.598 & 0.270 & 1843.340 & 1834.854 & 0.462 \\
\hline
\end{tabular}
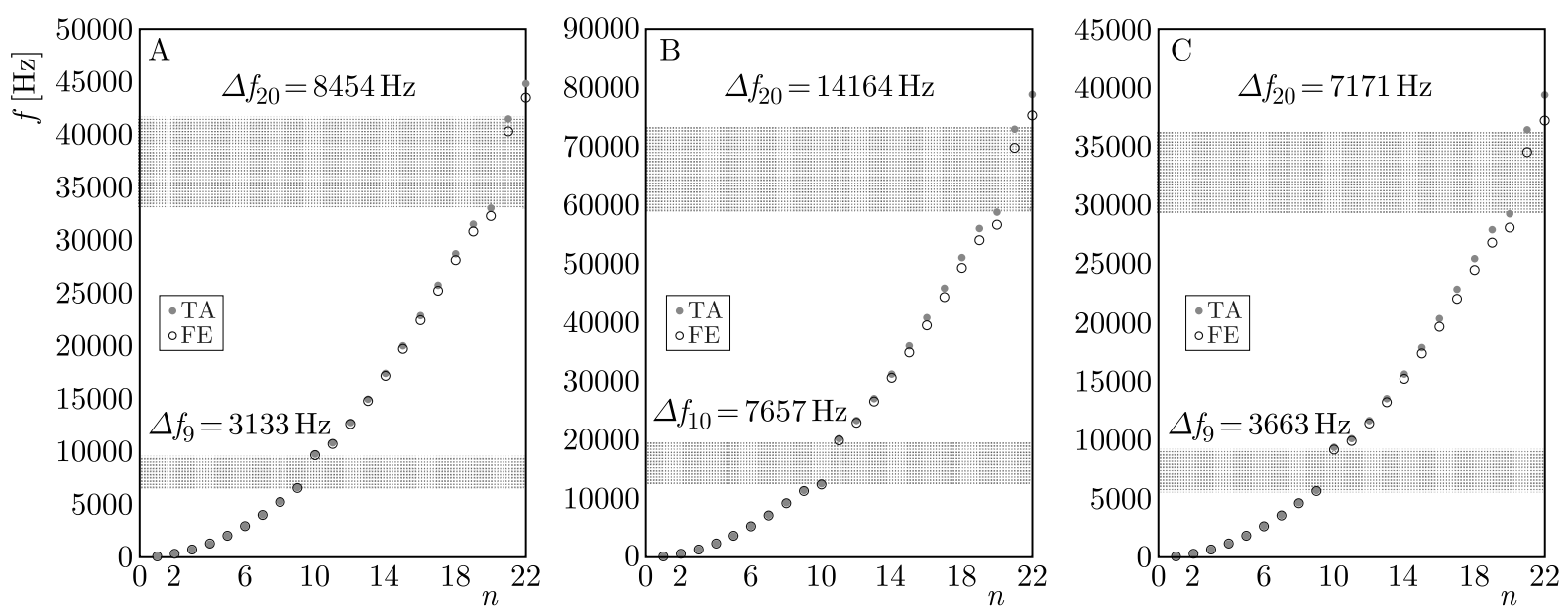

Fig. 3. Comparison of natural frequencies bandwidth of the considered beam for $\alpha=1 / 2$, case A2, B2, C2

In Figs. 4-6, the band gaps neighborhood is shown. The tolerance solutions are represented by solid lines, and the finite element solutions are represented by dashed lines. In this case, the presented frequencies are functions of the saturation parameter $\alpha$. All frequencies are presented in relation to the constant value - the natural frequency obtained from the finite element method. As a result, the solutions are presented in the dimensionless form. The first two gaps in the observed bandwidth range are analyzed. In cases $\mathrm{A}$ and $\mathrm{C}$, the analyzed frequencies are increasing with the argument of a function. In case B, a decreasing relationship can be observed. In the enclosed figures, two types of band gaps can be noticed. The first type of the gap is between the same frequencies in the entire domain of the mesostructure parameter. The second type of the gap changes its character along with the $\alpha$ parameter. The following relationship can be observed: the compared models have the best convergence for low natural frequencies and low values of the $\alpha$ parameter. 
A1

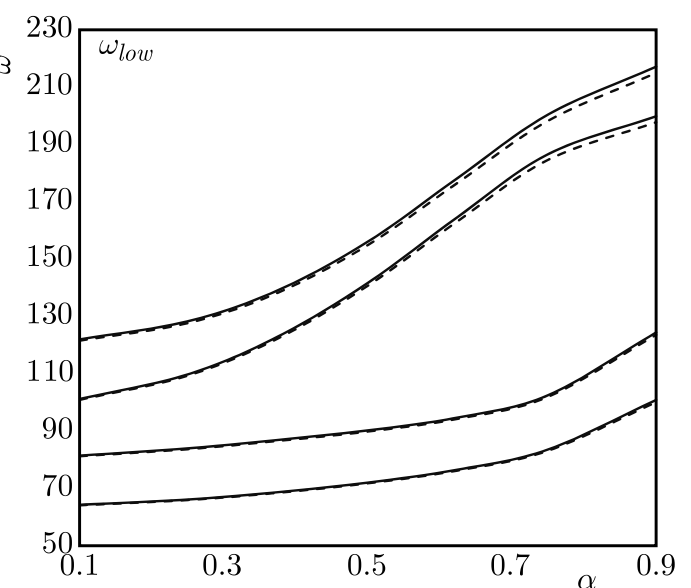

A2

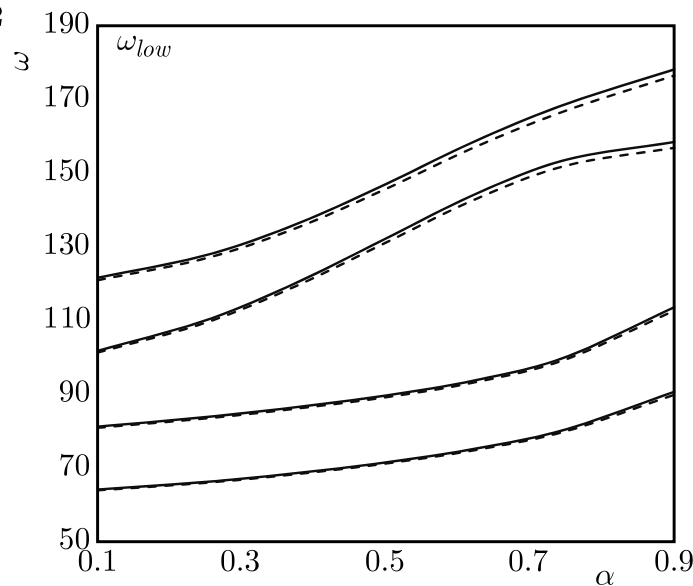

A3

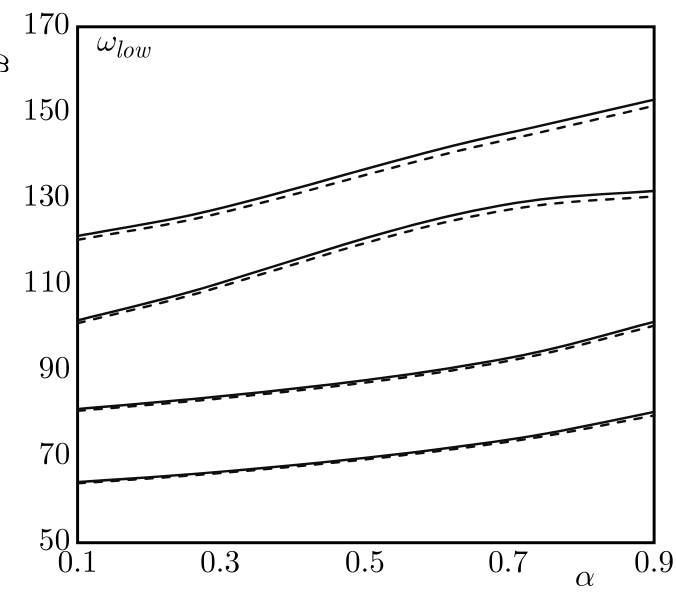

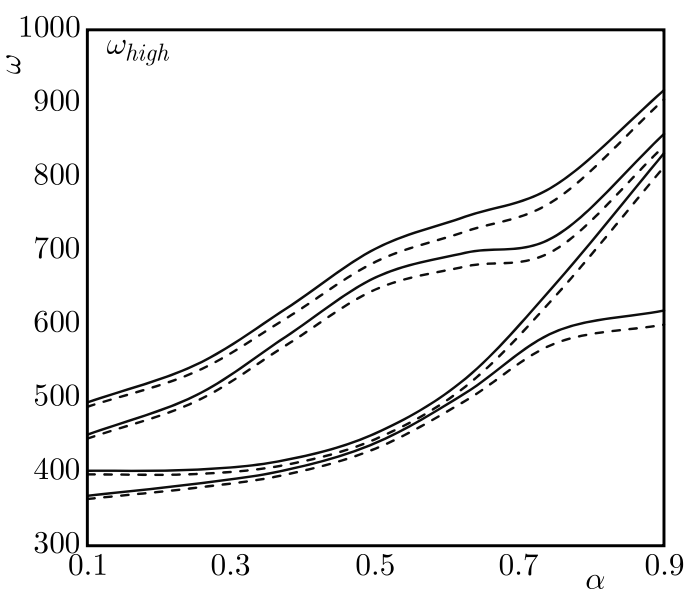
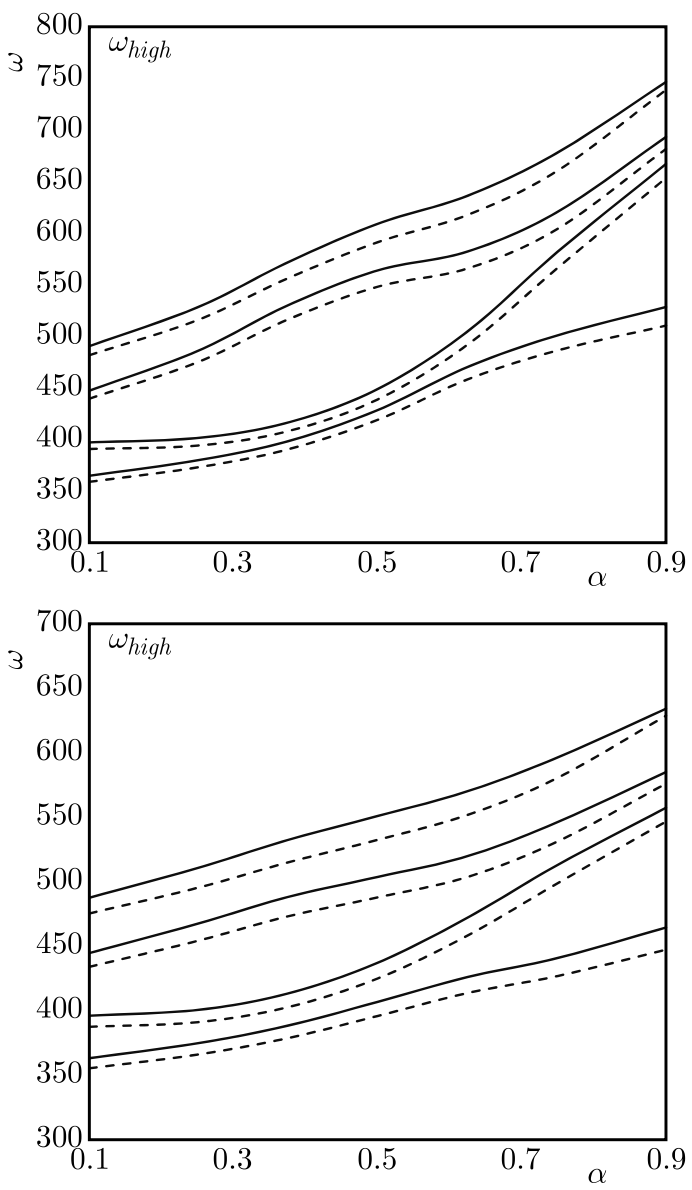

Fig. 4. Band-gap neighboring eigenfrequencies as a function of the $\alpha$ parameter, case A

In Table 3, the eigenmodes of the considered beam model for $\alpha=1 / 2$ are compared. It can be noticed that in B2 case the band gaps occur in different places in comparison with A2, $\mathrm{B} 2$ and $\mathrm{C} 2$ cases (Fig. 3). What is more, there is a difference in the order of symmetrical and antisymmetrical eigenmodes (cf. Table 3).

\section{Final remarks}

In this paper, the authors present a new averaged model of a linear-elastic periodic Rayleigh beam. Dynamics of the beam is described by partial differential equations with non-continuous 

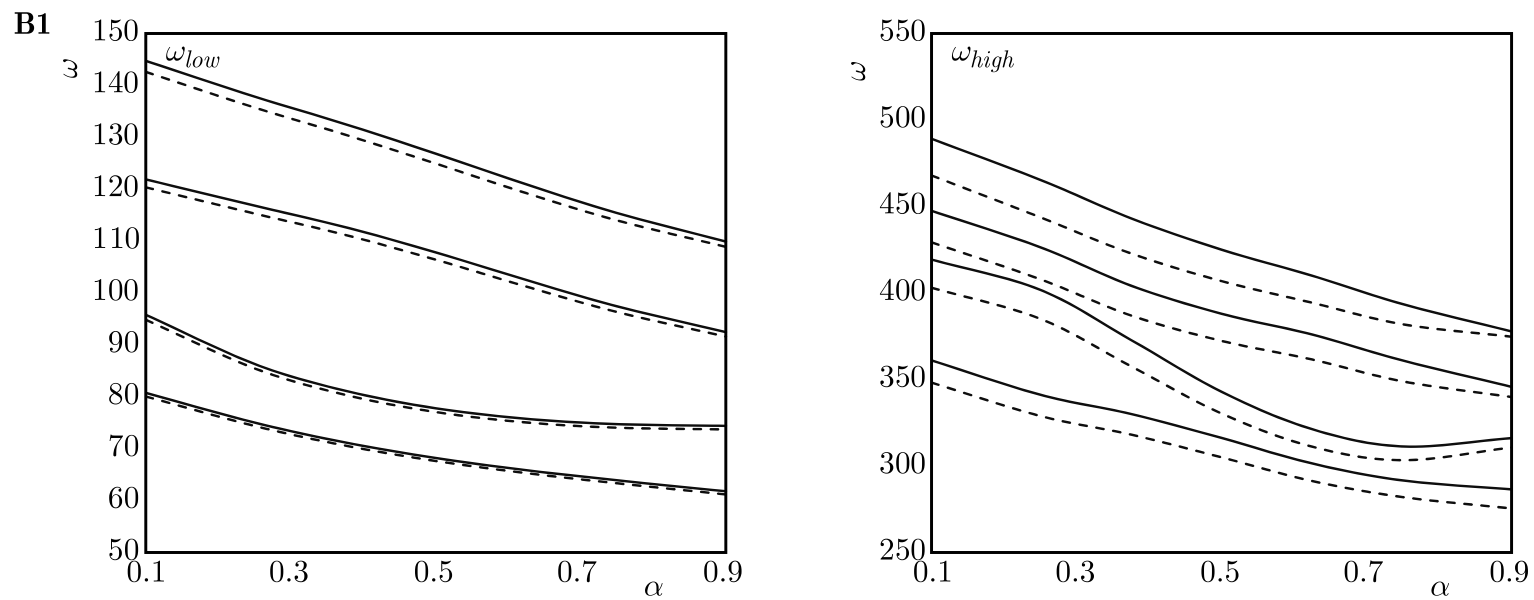

B2
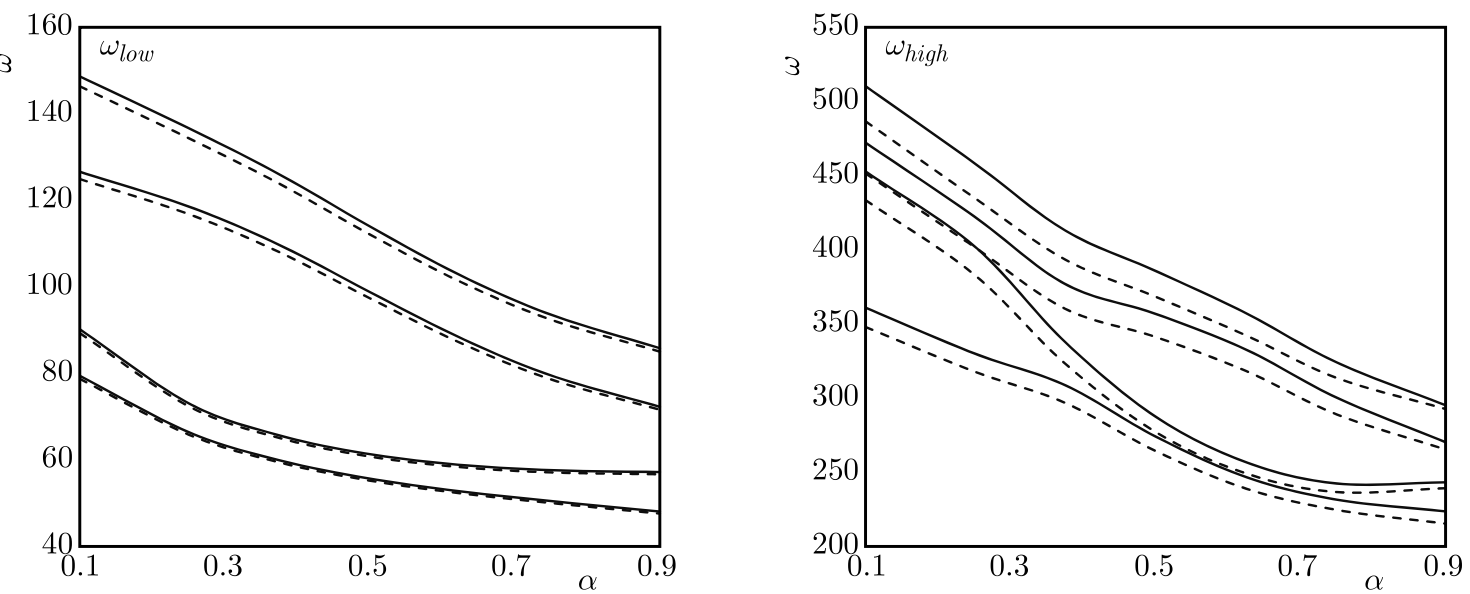

B3
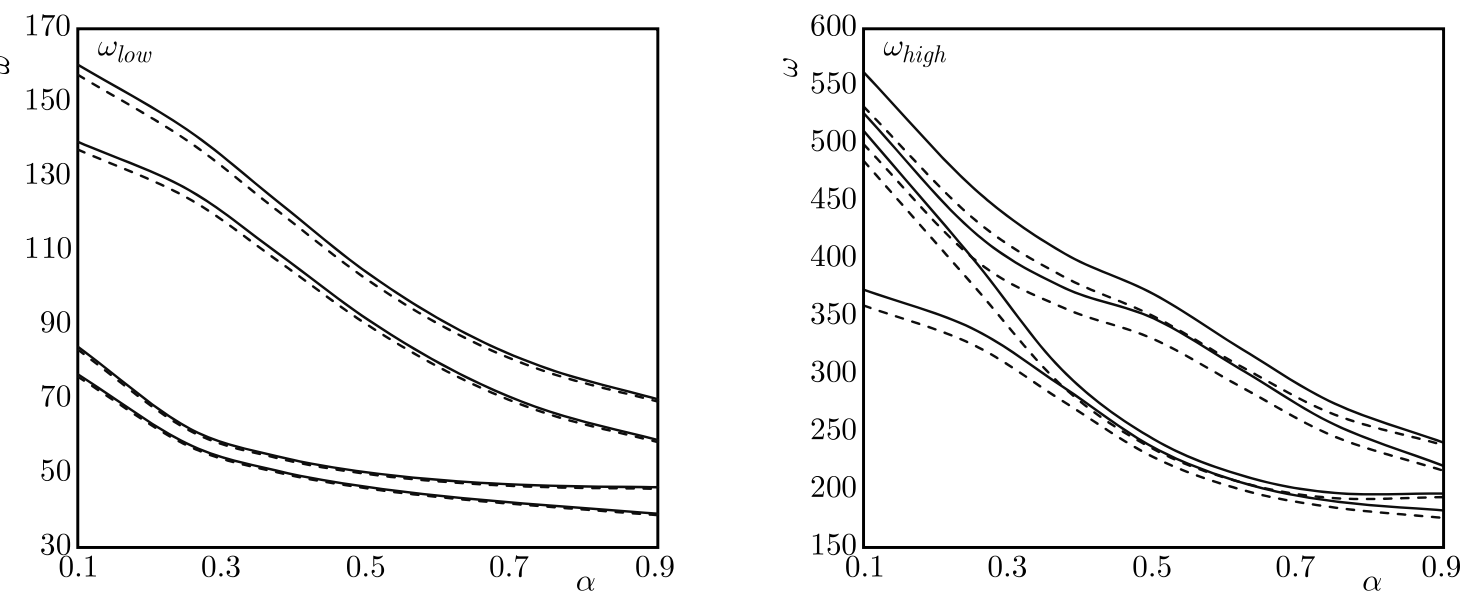

Fig. 5. Band-gap neighboring eigenfrequencies as a function of the $\alpha$ parameter, case B

highly oscillating coefficients. The exact model equations are transformed into a form that can be solved numerically.

The new model implements the notion of weakly slowly-varying functions. The proposed equations are derived using the tolerance averaging approach. In contradiction to other homogenized models, the tolerance averaging technique allows one to observe some averaged effective properties of a structure. Despite the inhomogeneity of the structure, this new model introduces some new unknowns - averaged deflection. It also allows one to observe some dynamic properties of the beam, depending on the size of the periodicity cell. 

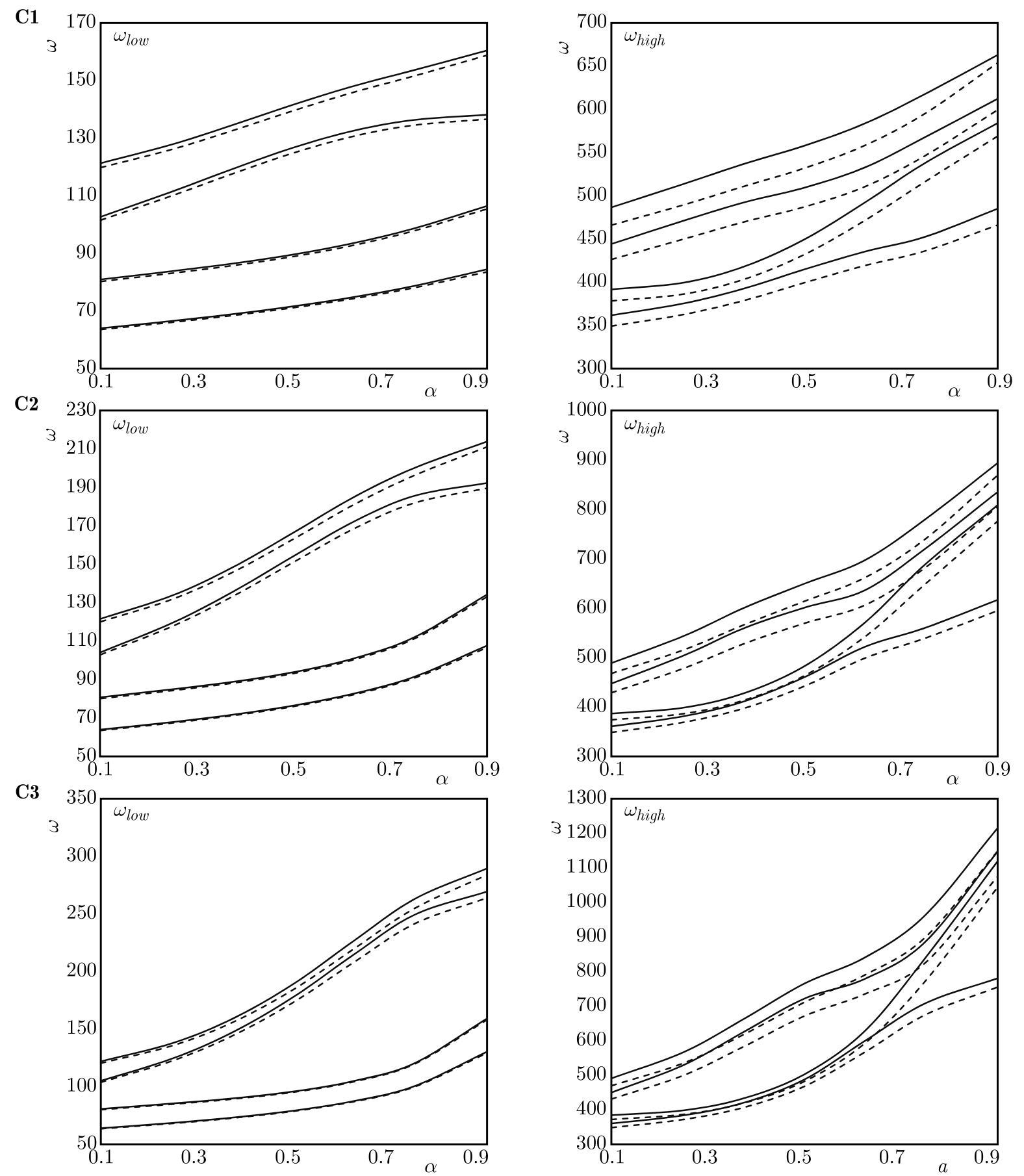

Fig. 6. Band-gap neighboring eigenfrequencies as a function of the $\alpha$ parameter, case $\mathrm{C}$

The solutions derived from tolerance averaging have been compared with the finite element model solutions. The finite element model has 31 nodes with 62 degrees of freedom.

In this paper, 3 cases have been compared: A, B and C. A good agreement has been obtained between the two methods in all analyzed cases. What is more, there is an evident dependency between the occurrence of band gaps and the shape of eigenmodes. Therefore, the proposed solution enables one to formulate model equations which can be solved with known numerical methods (e.g. Galerkin method). That is why the suggested technique can be used in the parametric analysis of the structures under consideration. The problems that can be considered in 
Table 3. Comparison of natural frequency bandwidths of the considered beam for $\alpha=1 / 2$

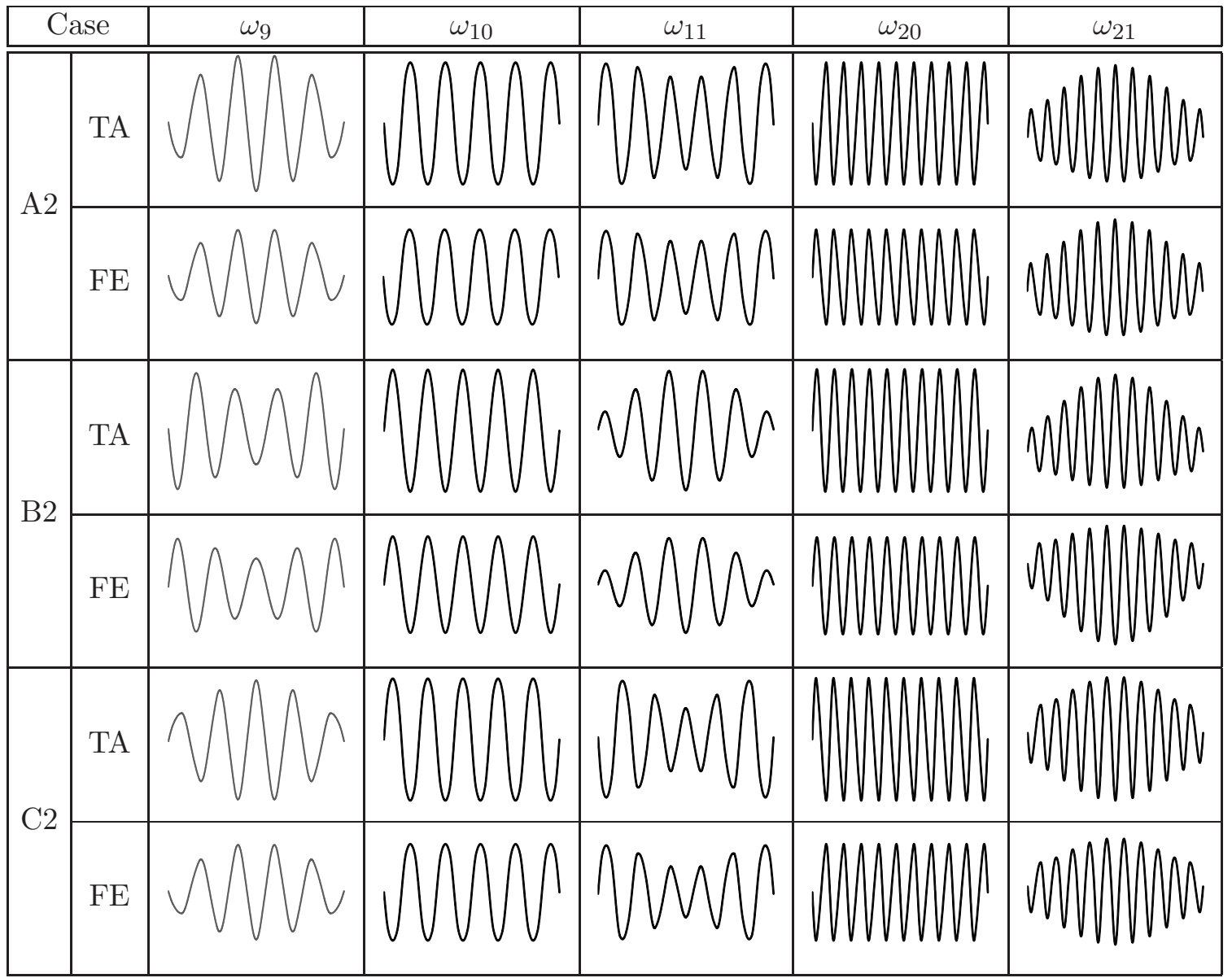

future works are: forced vibrations of inhomogeneous Rayleigh beams, greater diversity of boundary conditions, analysis of structural and material heterogeneity of the beam and a viscoelastic subsoil.

\section{Acknowledgements}

This contribution was supported by the National Science Centre of Poland under grant No. 2014/15/B/ST8/03155

\section{References}

1. Awrejcewicz J., 2010, Mathematical Modelling and Analysis in Continuum Mechanics of Microstructured Media: Professor Margaret Woźniak Pro Memoria: Sapiens Mortem Non Timet, Silesian University of Technology Publisher

2. Chen T., 2013, Investigations on flexural wave propagation of a periodic beam using multi-reflection method, Archive of Applied Mechanics, 83, 2, 315-329

3. Cielecka I., JęDrysiak J., 2006, A non-asymptotic model of dynamics of honeycomb lattice-type plates, Journal of Sound and Vibration, 296, 1, 130-149

4. Domagalski L., JęDRYsiaK J., 2015, On the tolerance modelling of geometrically nonlinear thin periodic plates, Thin-Walled Structures, 87 (Supplement C), 183-190

5. Domagalski L., JęDRYsiak J., 2016, Geometrically nonlinear vibrations of slender meso-periodic beams. The tolerance modeling approach, Composite Structures, 136, 270-277 
6. Fung Y., 1965, Foundations of Solid Mechanics, Prentice-Hall International Series in Dynamics, Prentice-Hall

7. Hajianmaleki M., QATu M.S., 2013, Vibrations of straight and curved composite beams: A review, Composite Structures, 100 (Supplement C), 218-232

8. JĘDrYsiak J., 2000, On the stability of thin periodic plates, European Journal of Mechanics A/Solids, 19, 3, 487-502

9. JĘDRYSIAK J., 2003, Free vibrations of thin periodic plates interacting with an elastic periodic foundation, International Journal of Mechanical Sciences, 45, 8, 1411-1428

10. JĘDRYSIAK J., 2013, Modelling of dynamic behaviour of microstructured thin functionally graded plates, Thin-Walled Structures, 71 (Supplement C), 102-107

11. JĘDRYSIAK J., 2014, Free vibrations of thin functionally graded plates with microstructure, Engineering Structures, 75 (Supplement C), 99-112

12. JĘDRYSIAK J., 2017, General and standard tolerance models of thin two-directional periodic plates, [In:] Shell Structures: Theory and Applications, Vol. 4, W. Pietraszkiewicz, W. Witkowski (Edit.), 101-104

13. JęDrysiak J., Michalak B., 2011, On the modelling of stability problems for thin plates with functionally graded structure, Thin-Walled Structures, 49, 5, 627-635

14. KAŹMierczAK M., JĘDrYsiaK J., 2011, Tolerance modelling of vibrations of thin functionally graded plates, Thin-Walled Structures, 49, 10, 1295-1303

15. Kohn R.V., Vogelius M., 1984, A new model for thin plates with rapidly varying thickness, International Journal of Solids and Structures, 20, 4, 333-350

16. Kolpakov A., 1991, Calculation of the characteristics of thin elastic rods with a periodic structure, Journal of Applied Mathematics and Mechanics, 55, 3, 358-365

17. Kolpakov A., 1998, Application of homogenization method to justification of 1-D model for beam of periodic structure having initial stresses, International Journal of Solids and Structures, 35, 22, $2847-2859$

18. Kolpakov A., 1999, The governing equations of a thin elastic stressed beam with a periodic structure, Journal of Applied Mathematics and Mechanics, 63, 3, 495-504

19. Mazur-ŚniAdy K., 1993, Macro-dynamic of micro-periodic elastic beams, Journal of Theoretical and Applied Mechanics, 31, 4, 781-793

20. Michalak B., 2001, The meso-shape functions for the meso-structural models of wavy-plates, ZAMM - Journal of Applied Mathematics and Mechanics / Zeitschrift für Angewandte Mathematik und Mechanik, 81, 9, 639-641

21. Papanicolau G., Bensoussan A., Lions J., 1978, Asymptotic Analysis for Periodic Structures, Studies in Mathematics and its Applications, Elsevier Science

22. SÁnchez-Palencia E., 1980, Non-Homogeneous Media and Vibration Theory, Lecture Notes in Physics, Springer-Verlag

23. TомсzYK B., 2013. Length-scale effect in dynamics and stability of thin periodic cylindrical shells, Research Bulletin, 466, 1-163

24. Woźniak C., Michalak B., JęDrysiak J., 2008, Thermomechanics of Microheterogeneous Solids and Structures: Tolerance Averaging Approach, Monografie - Lodz University of Technology, Lodz University of Technology Publisher

25. Woźniak C., WierzBicki E., 2000, Averaging Techniques in Thermomechanics of Composite Solids: Tolerance Averaging Versus Homogenization, Czestochowa University of Technology Publisher

26. XiAng H.-J., Shi Z.-F., 2009, Analysis of flexural vibration band gaps in periodic beams using differential quadrature method, Computers and Structures, 87, 23, 1559-1566 
27. Xu Y., Zhou X., Wang W., Wang L., Peng F., Li B., 2016, On natural frequencies of non-uniform beams modulated by finite periodic cells, Physics Letters A, 380, 40, 3278-3283

28. Yu D., Wen J., Shen H., Xiao Y., Wen X., 2012, Propagation of flexural wave in periodic beam on elastic foundations, Physics Letters A, 376, 4, 626-630

29. Zhikov V., Kozlov S., Olě̃nik O., 1994, Homogenization of Differential Operators and Integral Functionals, Springer-Verlag

30. Zienkiewicz O., Taylor R., Zhu J., 2013, The Finite Element Method: Its Basis and Fundamentals. The Finite Element Method, Elsevier Science

Manuscript received March 24, 2018; accepted for print August 8, 2018 